\title{
The relationship of estimated comprehensibility to the rate of connected speech
}

\author{
HENRY J. de HAAN \\ U.S. Army Research Institute, Alexandria, Virginia
}

\begin{abstract}
The relationship between subjective estimates of the comprehensibility of connected, freerunning speech and rate of speech was investigated for each of two types of time-compressed speech: pitch-varying speeded speech and pitch-normalized compressed speech. The midpoints of the resulting functions approximated the values obtained by a previously described speechrate tracking method. For equivalent degrees of comprehensibility, rates were higher for compressed speech than for speeded speech, indicating that estimates are sensitive to the intelligibility of speech. Subjective estimates of comprehensibility of time-compressed speech provide a means of assessing the intelligibility of connected speech.
\end{abstract}

The purpose of the present research is to investigate the relationship between the rate of connected, time-compressed speech and subjective estimates of its comprehensibility and to compare the method described here with a previously described method for measuring the speech-rate intelligibility threshold (de Haan, 1977).

In the past, investigations of the effects of rate of speech were hampered by the fact that the human speech production capacity is below the capacity for speech perception. Although the rate of speech can be increased by simply replaying a recording at a faster rate than it was originally recorded, such "speeded" speech suffers from increased pitch and decreased intelligibility. A method described by Garvey (1953) yields time-compressed speech with normal pitch (compressed speech). Technological developments during the last 15 years have led to improved electronic speech compressors based on this method. These devices electronically discard brief portions of the speech signal on a periodic basis that is independent of any particular phonemic component of the speech. The brevity of the discard interval precludes the loss of entire phonemes, although there are large decreases in phoneme duration at extremely high speech rates.

Methods for the evaluation of time-compressed speech have been reviewed previously (Foulke \& Sticht, 1969). Two characteristics of compressed

This work was presented in part at the 86th Annual Convention of the American Psychological Association, Toronto, Canada, August 28-September 1, 1978. Requests for reprints should be sent to Henry J. de Haan, U.S. Army Institute for the Behavioral and Social Sciences, 5001 Eisenhower Avenue, Alexandria, Virginia 22333. The views expressed in this paper are those of the author and do not necessarily represent those of the Department of the Army or the U.S. Army Research Institute. The designation of commercial products does not constitute official endorsement of them by either of the above. speech that are frequently measured are speech intelligibility and comprehensibility. Intelligibility refers to the recognition of words or phonemes and implies information processing at the phonemic level. The percentage of words correctly identified from a word list is usually taken as the index of intelligibility. In 1953, Garvey not only developed the sampling method of speech compression described above, but also applied the intelligibility test to compressed words. His results specified the intelligibility of both speeded and compressed words as a function of rate of speech and showed that compressed words were more intelligible than speeded words.

Comprehensibility or comprehension-the choice of terms depends on whether the emphasis is on properties of speech or psychological processes and individual differences-refers to understanding the meaning of language and implies information processing at the semantic level. Comprehensibility is assessed by the application of the comprehension test to connected discourse or free-running speech. The index of comprehensibility is the percentage of correct answers to questions that concern the content of a passage of connected discourse. The comprehension test has been used to investigate the effect of rate of speech on comprehensibility. Foulke and Sticht (1969) reported that comprehension declines relatively slowly up to a word rate of 275 words per minute (wpm) but more rapidly beyond that point. This statement implied that there was a threshold of comprehension as a function of word rate.

Subsequently, a subjective threshold method was developed to determine this rate threshold (de Haan, 1977; de Haan \& Schjelderup, 1978). It is analogous to the Békésy (1947) method for measuring intensity thresholds; however, rate, rather than intensity, of speech is varied. Rate of speech increases automatically until the listener presses a button indicating that speech can no longer be understood. The rate of 
speech then decreases until the listener releases the button indicating that speech can again be understood. A repetition of this process, in which the listener responds to and controls the changing rate of speech, effectively brackets the speech rate at which understanding fails. This rate-tracking threshold is based on only two response categories: listeners judge that they either do or do not understand speech at the moment of response.

This threshold has been called an intelligibility threshold and an intelligibility/comprehensibility threshold. The threshold did reflect intelligibility. It gave differential results with two types of timecompressed speech known to differ in intelligibility: simple speeded speech and compressed speech (Garvey's sampling method). The mean threshold value of compressed speech also appeared to be near the word rate at which the rapid decline in comprehension occurs when measured by comprehension test scores, implying that it measured comprehensibility. In spite of this, thresholds were not highly correlated with comprehension test scores, perhaps, in part, because moderate comprehension test reliabilities attenuated the correlations (de Haan, 1977).

While intelligibility and comprehension tests are objective measures, the rate-tracking threshold involves a subjective measure. There are examples in the literature of the use of subjective methods for measuring both intelligibility and comprehensibility. Subjective measures of comprehensibility are exemplified by the work of Schwartz, Sparkman, and Deese (1970) and by the work of Wang (1970a, 1970b). These investigators required listeners to make subjective judgments of the comprehensibility of isolated sentences and reported that their measures could be validated either against the syntactic and structural complexity of sentences or against readability indices.

Subjective methods have also been applied to the measurement of intelligibility of connected, freerunning speech (Chaiklin, 1959; Dahle, Hume, \& Haspiel, 1968; Falconer \& Davis, 1947; Haspiel \& Havens, 1966; Hawkins \& Stevens, 1950; LeZak, Siegenthaler, \& Davis, 1964; Speaks, Parker, Harris, \& Kuhl, 1972). These investigators required the listeners to adjust the intensity of faint speech until they could just understand it or, in the case of Speaks et al., understand a given percentage of it. Several of the investigators used the Békésy technique for the determination of speech intensity thresholds and referred to the latter as speech-Békésy thresholds. All of these experiments were concerned with the understanding of speech as a function of its intensity rather than with its rate, but it should be emphasized that they used subjective methods to measure intelligibility.

In general, the purpose of the present work was to investigate the functional relationship between degree of estimated comprehensibility and rate of free-running, connected, time-compressed speech. Additional objectives included the comparison of hypothesized functions for two types of time-compressed speech known to differ in intelligibility and the comparison of rates at midpoints of the functions with values of speech-rate thresholds determined by the previously described rate-tracking method.

\section{METHOD}

\section{Participants}

Thirty-two military enlisted personnel (22 males and 10 females) participated in this research. With three exceptions, personnel had general ability test scores (Armed Services Vocational Aptitude Battery, General Technical Score) of 100 or above. The group mean was 116.22 and the standard deviation was 14.38. None of the participants had had previous experience with compressed speech.

\section{Apparatus}

Apparatus consisted of a variable-speed tape recorder (Crown 800 Series), an AmBiChron speech compressor/expander (Koch, 1974), a strip-chart recorder, a frequency counter, and a laboratoryfabricated ramp generator (to accelerate or decelerate speech). Speech was delivered binaurally through headphones. Rate of speech was controlled either by a 10-turn potentiometer knob or, in the case of rate-tracking threshold determinations, by a pushbutton switch (yielding either acceleration or deceleration of speech). A laboratory computer (ADS 1800E) was used to regulate the sequence of events during threshold determinations and to record and average responses. This apparatus is described more fully elsewhere (de Haan and Schjelderup, 1978).

\section{Materials}

Stimulus materials were passages of speech selected from a Library of Congress Talking Book (tape recording), The Proud Tower (Tuchman, 1966). The passages were read at an average rate of 126 wpm by a female professional reader. These materials are described more fully elsewhere (de Haan, 1977).

\section{Design and Procedure}

Listeners were individually presented with connected speech through earphones at a comfortable listening level. The rate of speech could be adjusted by a control knob. Listeners were asked to set speech rates to correspond to several predesignated percentages estimated to be understood. These percentages, specified by the experimenter, were presented visually in the following order: $50,75,25,90,10,100,0,50,25,75,10,90,0$, and 100 . This sequence involved two judgments at each of the following percentages: $0,10,25,50,75,90$, and 100 . Both speeded and compressed speech were presented to each listener, half of the listeners receiving speeded speech first and the other half compressed speech first.

A modified Latin square experimental design was used (Winer, 1971, pp. 745-746). The main independent variables, specified percentage and type of speech, were within-subject variables. Practice effect was also a within-subject variable. Order of presentation (speeded vs. compressed speech first) was a between-subjects variable.

For comparison with rate-setting data, rate-tracking thresholds for both speeded and compressed speech were determined for each individual by the method described earlier (de Haan, 1977; de Haan \& Schjelderup, 1978). This method required listeners to press a button when they estimated that they could no longer understand speech as it accelerated at a constant rate. Their response caused the speech to decelerate at a constant rate. Listeners were instructed to release the button when they estimated that they could again understand it. A repetition of this process 
yields a threshold value. Four 3-min thresholds were determined per listener, each at different rates of acceleration-deceleration, and the means of the four determinations constituted the listener's threshold. Additional details of the method can be found in the earlier papers.

\section{RESULTS}

In Figure 1, estimated comprehensibility is plotted on the ordinate and rate of speech (relative to the original speech rate), on the abscissa.

Figure 1 shows an orderly relationship between estimated comprehensibility and rate of speech. The effect of percentage is significant $[F(6,180)=184.57$, $\mathrm{p}<.01$ ]. In general, compressed speech is estimated to be comprehensible at a higher rate of speech than is speeded speech $[F(1,30)=273.27, p<.01]$. An interaction between percentage and type of speech may be seen as an increasingly greater divergence between the curves at the lower percentages $[F(6,180)$ $=82.13, \mathrm{p}<.01]$. The differences between the functions for speeded and compressed speech are probably due to the fact that the intelligibility of speeded speech is lower than that of compressed speech and becomes increasingly so as rate increases. A practice effect was also observed $[F(1,30)=5.44, p<.05]$. Order of presentation (speeded vs. compressed first) was not significant $[F(1,30)=.79, p>.05]$.

An estimate of the reliability of the data was obtained by correlating the sum of the first trial responses (one at each of seven percentages) with the sum of the second trial responses. The values of the correlation coefficients were .908 and .954 for speeded and compressed speech, respectively. The values obtained by correlating single responses (Trial 1 vs.

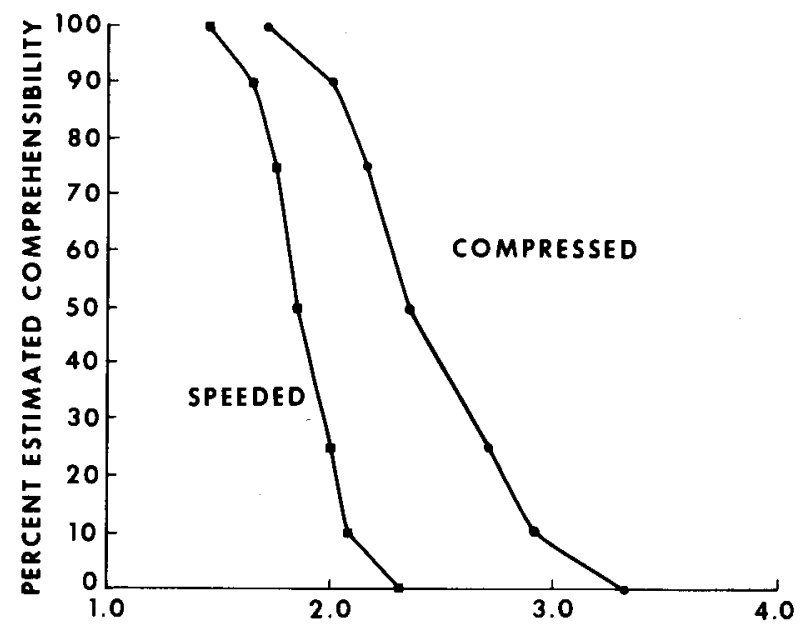

SPEECH RATE IN MULTIPLES OF THE ORIGINAL RATE

Figure 1. The relationship of entimated comprehensibility to the rate of connected speech. The curves are for free-running speech time-compressed by two different methods.
Table 1

Means and Standard Deviations (SD) of Speech-Rate Tracking Thresholds and Midpoints of Rate-Setting Functions in Relative Rates of Speech

\begin{tabular}{|c|c|c|c|c|c|c|}
\hline & \multicolumn{6}{|c|}{ Type of Speech } \\
\hline & \multicolumn{3}{|c|}{ Speeded } & \multicolumn{3}{|c|}{ Compressed } \\
\hline & Mean & $\mathrm{SD}$ & WPM & Mean & SD & WPM \\
\hline $\begin{array}{l}\text { Mean Tracking } \\
\text { Threshold }\end{array}$ & 1.79 & .173 & 226 & 2.41 & .342 & 304 \\
\hline $\begin{array}{l}\text { Mean Rate-Setting } \\
\text { Midpoint }(50 \%)\end{array}$ & 1.84 & .170 & 232 & 2.35 & .365 & 296 \\
\hline
\end{tabular}

Note-WPM $=$ words per minute.

Trial 2 at each estimated percentage) were, of course, lower. The average values (averaged by Fisher's Z) of the coefficients were .825 (range: .691 to .933 ) and .870 (range: .812 to .911 ) for speeded and compressed speech, respectively.

Comparisons were made between mean speech rates at the midpoints of the estimated comprehensibility functions and mean thresholds determined by the rate-tracking method developed earlier (de Haan, 1977). Table 1 shows the mean rates at the midpoints of the functions ( $50 \%$ estimated comprehensible) and mean rate-tracking thresholds in relative rates of speech (expressed as multiples of the original speech rate). Mean wpm also is shown. The mean rates at the midpoints of the estimated comprehensibility functions and the mean rate-tracking threshold values were not significantly different for either speeded $[\mathrm{t}(31)=1.8, \mathrm{p}>.05]$ or compressed speech $[t(31)=.82, p>.05]$. Even though the differences are not statistically significant, their importance is magnified by the steepness of the curves. Consequently, the methods yield similar, but not identical, results.

\section{DISCUSSION}

The main purpose of the present investigation was primarily empirical, to determine whether there is a functional relationship between estimated comprehensibility and rate of speech. The results indicate that there is such a relationship for both types of time-compressed speech. The data are orderly and show considerable reliability.

In the earlier work on estimated comprehensibility (de Haan, 1977), a speech-rate threshold was described which was based on only two categories of judgment: as the speech rate increased, listeners judged when they could no longer understand speech, and as the speech rate decreased, they judged when they could again understand it. Although, the average of these rates yielded a threshold value, that work could not reveal a complete functional relationship. Thus, the present work may be viewed as an extension of the earlier work. 
Since the midpoint of the functions also represents a speech-rate threshold, one of the purposes of the present work was to compare the rate-tracking method previously described with the rate-setting method employed here. The analysis revealed that the two methods yielded similar, if not equivalent, results.

It is interesting to note that the value of the midpoint of the compressed speech function-approximately $300 \mathrm{wpm}$-is roughly comparable to the word rate at which comprehension is said to decline when measured by objective comprehension tests, approximately 275 wpm (Foulke \& Sticht, 1969). Heiman and Leighbody (Note 1), who compared interrupted and compressed speech with similar deletion parameters, also found that comprehension of compressed speech declined rapidly at rates roughly similar to those in the present investigation (between 2.0 to 2.3 times the original rate of speech in their work). Even so, the similarity between subjective and objective measures of comprehensibility does not imply that identical processes are involved, particularly since the earlier work (de Haan, 1977) did not find a high relationship between the measures.

Comparison of the functions for speeded and compressed speech revealed that, for each estimated percentage comprehensible, the rate was higher for compressed than for speeded speech. Wallace and Koury (1981) found that compressed sentences were more intelligible than speeded sentences when intelligibility was assessed by the proportion of words recalled. (This work also revealed that both increased pitch and increased word rate contributed to intelligibility decrements.) Since intelligibility is a precondition for comprehensibility, estimates of comprehensibility may also be dependent on intelligibility. This would account for the differences between speeded and compressed estimates in the present investigation.

While the present work is primarily empirical, a theoretical explanation of the above findings may be offered. Postman, Thompkins, and Gray (1978) have stated that subjects approach an experimental situation with a strong tendency to process words semantically, and that this disposition cannot be entirely overridden by instructions to process information nonsemantically. According to their views, a nonsemantic task can interfere with, but not completely eliminate, this tendency for semantic processing.

These views may be applicable to the difference between speeded and compressed speech functions in the present investigation. A listener who is exposed to connected (time-compressed) speech has a tendency to process the speech semantically. However, the unusual nature of the speech signal may require some processing on a nonsemantic level. This is particularly true of speeded speech, since its intelligibility in comparison with that of compressed speech is reduced by higher pitch. As a result, a relatively greater amount of phonemic processing may be required. Processing at the phonemic level may interfere with processing at the semantic level and, to the extent that phonemic processing is required, both comprehension and estimated comprehensibility will be reduced. Consequently, for equivalent degrees of estimated comprehensibility, the rate will be lower for speeded than for compressed speech.

The fact that comprehensibility estimates of timecompressed speech are sensitive to speech intelligibility provides a means of assessing the intelligibility of connected speech. This is the rationale behind the previously described speech-rate intelligibility threshold, and the present work provides an alternative method for its determination. The present work also points to the need for further investigations into the similarities and differences between estimated and measured comprehensibility and the processes that underlie them.

\section{REFERENCE NOTES}

1. Heiman, G. W., \& Leighbody, G. P. Comprehension decrements in compressed speech: Intelligibility decrements or processing overload. Paper presented at the 86th Annual Meeting of the American Psychological Association, Toronto, Canada, September 1978.

\section{REFERENCES}

BÉkÉsy, G. von. A new audiometer. Acta Otolaryngologica, $1947,35,411-422$.

Chaiklin, J. B. The relation among three selected auditory speech thresholds. Journal of Speech \& Hearing Research, 1959, 2, 237-243.

Dahle, A. J., Hume, W. C., \& Haspiel, G. S. Comparison of speech-Békésy tracings with selected clinical auditory measures. Journal of Auditory Research, 1968, 8, 125-128.

DE HAAN, H. J. A speech-rate intelligibility threshold for speeded and time-compressed connected speech. Perception \& Psychophysics, 1977, 22, 366-372.

de HaAN, H. J., \& SchjeldenuP, J. R. Threshold of intelligibility/comprehensibility of rapid connected speech: Method and instrumentation. Behavior Research Methods \& Instrumentation, 1978, 10, 841-844.

Falconer, G., \& Davis, H. The intelligibility of connected discourse as a test for the threshold of speech. Laryngoscope, $1947,57,581-595$.

Foulke, E., \& Sticht, T. G. A review of research on timecompressed speech. Psychological Bulletin, 1969, 72, 50-62.

GARVEY, W. D. The intelligibility of speeded speech. Journal of Experimental Psychology, 1953, 54, 102-108.

Haspies, G., \& Havens, R. Comparison of speech-Békésy with pure tone Békésy thresholds. Journal of Auditory Research, 1966, 6, 235-237.

Hawkins, J. E., \& Stevens, S. S. The masking of pure tones and of speech by white noise. Journal of the Acoustical Society of America, 1950, 22, 6-13.

Koch, R. The AmBiChron. In S. Duker (Ed.), Time compressed speech: An anthology and bibliography. Metuchen, N.J: Scarecrow Press, 1974.

LeZak, R. K., Siegenthaler, B. R., \& Davis, A. J. Békésytype audiometry for speech reception threshold. Journal of Auditory Research, 1964, 4, 181-190.

Postman, L., Thomprins, B. A., \& Gray, W. D. The interpretation of encoding effects in retention. Journal of Verbal Learning and Verbal Behavior, 1978, 17, 681-705. 
Schwartz, D., Sparkman, J. P., \& Deese, J. The process of understanding and judgments of comprehensibility. Journal of Verbal Learning and Verbal Behavior, 1970, 9, 87-93.

Speaks, C., Parker, B., Harris, C., \& Kuhl, P. Intelligibility of connected discourse. Journal of Speech \& Hearing Research, 1972, 15, 590-602.

Tuchman, B. The proud tower. New York: Macmillan, 1966.

Wallace, W. P., \& Koury, G. Transfer effects from listening to frequency-controlled and frequency-shifted accelerated speech. Journal of Speech and Hearing Research, 1981, 24, 185-191.
WANG, M. D. The role of syntactic complexity as a determiner of comprehensibility. Journal of Verbal Learning and Verbal Behavior, 1970, 9, 398-404. (a)

WANG, M. D. Influence of linguistic structure on comprehensibility and recognition. Journal of Experimental Psychology, 1970, 95, 83-89. (b)

Winer, B. J. Statistical principles in experimental design (2nd ed.). New York: McGraw-Hill, 1971.

(Manuscript received August 17, 1981; revision accepted for publication April 12, 1982.) 